\section{Original Article}

Check for updates

\section{OPEN ACCESS}

Received: Jan 18, 2019

Revised: Mar 18, 2019

Accepted: Mar 18, 2019

Correspondence to

Yoo Seung Chung

Department of Surgery, Gil Medical Center, Gachon University College of Medicine, 21

Namdong-daero 774 beon-gil, Namdong-gu, Incheon 21565, Korea.

E-mail: dryooseung@gilhospital.com dryooseung@hanmail.net

Copyright (c) 2019. Korean Association of Thyroid and Endocrine Surgeons; KATES This is an Open Access article distributed under the terms of the Creative Commons Attribution Non-Commercial License (https:// creativecommons.org/licenses/by-nc/4.0/).

ORCID IDs

Joon-Hyop Lee (iD)

https://orcid.org/0000-0003-0470-7719

Yoo Seung Chung (D)

https://orcid.org/0000-0001-9912-051X

Conflict of Interest

No potential conflict of interest relevant to this article was reported.

Author Contributions

Conceptualization: Yoo Seung Chung. Data curation: Shin Dol Jo. Investigation: JoonHyop Lee. Supervision: Yoo Seung Chung. Writing - original draft: Shin Dol Jo. Writing review \& editing: Suk Ha Kang, Yun Yeong Kim, Yong Soon Chun, Heung Kyu Park, Sang Tae Choi, Jin Mo Kang, Yoo Seung Chung.

\title{
Risk Factors for Distant Metastasis in Follicular Thyroid Carcinoma in Korea
}

\author{
Shin Dol Jo', Joon-Hyop Lee $\mathbb{1}^{1,2}$, Suk Ha Kang', Yun Yeong Kim ${ }^{1,2}$, \\ Yong Soon Chun ${ }^{1,2}$, Heung Kyu Park ${ }^{1,2}$, Sang Tae Choi ${ }^{1,2}$, Jin Mo Kang ${ }^{1,2}$, \\ Yoo Seung Chung $\left.{ }^{1}\right)^{1,2}$
}

'Department of Surgery, Gil Medical Center, Incheon, Korea

${ }^{2}$ Department of Surgery, Gachon University College of Medicine, Incheon, Korea

\section{ABSTRACT}

Purpose: Follicular thyroid cancer (FTC) can frequently metastasize to distant organs, which worsens the prognosis. This study evaluated the risk factors of distant metastasis in FTC. Methods: We retrospectively reviewed 149 patients diagnosed with primary FTC and underwent operations between 2001 and 2017.

Results: The mean age of all patients was $44.8 \pm 14.4$ years and $82.6 \%$ were female. The mean tumor size was $3.44 \pm 1.77 \mathrm{~cm}$ and a total thyroidectomy was performed in $41.6 \%$ of cases. The follow-up duration was $65.4 \pm 43.5$ months. Distant metastases occurred in 8 patients (5.4\%): 5 patients had synchronous and 3 had metachronous distant metastasis. The most common metastatic site was the bone, with bone metastasis observed in 5 of 8 patients, followed by the lung ( 3 of 8 ). Bone metastases occurred in 4 of 5 patients with synchronous distant metastasis and in 1 of 3 with metachronous distant metastasis. Distant metastasis was associated with old age ( $\geq 45$ years), the presence of vascular invasion, widely invasive FTC, and high thyroglobulin (Tg) levels in the first postoperative month. Multivariate analysis showed that old age and the presence of vascular invasion were predictive factors for distant metastasis $(\mathrm{P}=0.016$ and $\mathrm{P}=0.013$, respectively).

Conclusion: Old age and vascular invasion were significant factors for distant metastasis. The possibility of bone and lung distant metastases should be assessed when a patient is old ( $\geq 45$ years), FTC is widely invasive, and when Tg levels are high in the first postoperative month.

Keywords: Follicular thyroid carcinoma; Metastasis; Prognostic factor; Age of onset; Prognosis

\section{INTRODUCTION}

Follicular thyroid cancer (FTC) is the second most common thyroid cancer and constitutes approximately $10 \%$ of all thyroid malignancies (1). In Korea, FTC accounted for $1.6 \%$ of all thyroid cancers in 2015 (2). FTC can only be confirmed by pathological examination after diagnostic thyroidectomy and not by fine-needle aspiration cytology or frozen sections. Histologic diagnosis of FTC is based on the demonstration of follicular cells occupying abnormal positions, including capsular and/or vascular invasion. Minimally invasive FTCs have limited capsular and/or vascular invasion. Widely invasive FTCs have widespread 
infiltration of adjacent thyroid tissue and/or blood vessels (3). FTC typically spreads via hematogenous routes which occurs in $10 \%$ to $15 \%$ of cases with metastases to distant organs, most commonly involving the lung followed by bone rather than the lymph nodes. The brain, liver, and skin are less common sites of systemic metastases $(1,4)$.

The prognoses for FTC are worse than those for papillary thyroid carcinomas (PTC). In differentiated thyroid carcinoma, follicular histology is a significant factor for survival, and the 3-year survival rate for papillary and follicular carcinomas with distant metastases was $75 \%$ and $62 \%$, respectively, in the study of Canadian patients (5). In another study, the follicular histologic type of thyroid cancer also showed a significantly worse prognosis. The 5-year PTC survival rate with distant metastasis was $87.9 \%$ and $71.7 \%$ for FTC in the study of Korean patients (6). Furthermore, the survival rate was worse in cases of FTC with distant metastasis, while FTC without distant metastasis is a non-aggressive disease with a good prognosis (6-10).

It is our opinion that predicting the occurrence of metastases and assessing distant metastases are very important in managing FTC patients. Therefore, we performed this study to evaluate the clinicopathologic factors associated with distant metastases and considered effective methods for managing FTC patients.

\section{MATERIALS AND METHODS}

A total of 5,522 thyroid carcinoma operations were performed between 2001 and 2017. In the same period, 149 primary FTC patients underwent operations, and in this study, we retrospectively reviewed the medical records of these patients.

The diagnosis of FTC was made by postoperative histologic confirmation of primary thyroid tissue or distant metastatic organ tissues. We diagnosed minimally invasive FTCs as having limited capsular and/or vascular invasion and categorized widely invasive FTCs as having widespread infiltration into adjacent thyroid tissue and/or blood vessels ( $>4$ foci of vascular invasion) (3).

Initially, we performed a thyroid lobectomy and then a completion thyroidectomy after confirmation of the final diagnosis. Completion thyroidectomy was performed when the tumor size was $>4 \mathrm{~cm}$, if the FTC was widely invasive, or when multiplicity was confirmed. We could not perform a second operation if the patients refused to undergo another surgery. A total thyroidectomy was performed during the first operation in the case of accompanying bilateral indeterminate thyroid nodules, gross extra-thyroidal extension with soft tissue invasion, distant metastasis, or at the request of the patient.

Postoperative ${ }^{131}$ I radioactive iodine (RAI) therapy was applied to patients who had distant metastasis, tumors $>4 \mathrm{~cm}$, or widely invasive FTC. However, RAI therapy could not be performed if patients refused RAI therapy or if underlying diseases were severe.

Distant metastasis was verified using a ${ }^{131}$ I whole body scan (WBS), chest computed tomography (CT), bone scans, ${ }^{18} \mathrm{~F}$-fluorodeoxyglucose positron emission tomography-CT $\left({ }^{18} \mathrm{FDG}\right.$ PET-CT), or histologic confirmation of metastatic lesions. Chest CTs, bone scans, and PET-CTs were used to determine the presence of distant metastasis in patients with thyroid 
stimulating hormone (TSH) suppression whose thyroglobulin (Tg) level was consistently increasing during the follow-up period. Synchronous distant metastasis was defined as a metastasis that was detected during the initial diagnosis or within 12 months of the initial operation. Metachronous distant metastasis was defined as a metastasis found $\geq 12$ months after the initial operation. We analyzed synchronous and metachronous distant metastases together because of the small number of patients with metastases.

Our Institutional Review Board approved this retrospective study before the patient list was retrieved from the hospital database (GAIRB2018-429).

Statistical analysis was performed using IBM SPSS version 23.0 (SPSS Inc., Chicago, IL, USA). Pearson's $\chi^{2}$ test, Fisher's exact test, and the independent t-test were used to evaluate the significant differences between patients with and without distant metastasis. Logistic regression analyses were performed for multivariate analyses. P values $<0.05$ were considered statistically significant.

\section{RESULTS}

\section{Clinicopathologic characteristics of all 149 FTC patients}

The mean age at diagnosis was $44.8 \pm 14$. 4 years. The proportion of patients $<45$ years of age was $52.3 \%$ and $<55$ years of age was $75.8 \%$. There were 15 cases of widely invasive FTCs. A total thyroidectomy was performed in $41.6 \%$ of the patients. There were three cases of distant recurrences $(2.01 \%)$. The mean time to recurrence was $73.7 \pm 62.7$ months (34-176). A total of 8 patients suffered from distant metastases (5.4\%); 5 being synchronous and 3 metachronous. Metastatic lesions in synchronous metastases included 4 in bone and 1 in the lung and for metachronous metastases, there was 1 in bone and 2 in the lung. The mean serum $\mathrm{Tg}$ level in the first postoperative month was $54.6 \pm 93.8 \mathrm{ng} / \mathrm{mL}$ and the $\mathrm{Tg}$-antibody (Tg-Ab) level was 4.6 $\pm 14.9 \mathrm{U} / \mathrm{mL}$. The mean serum $\mathrm{Tg}$ level in the third postoperative month was $11.8 \pm 51.4 \mathrm{ng} / \mathrm{mL}$ and the $\mathrm{Tg}$-Ab level was $4.0 \pm 11.8 \mathrm{U} / \mathrm{mL}$. The follow-up duration was 65.4 \pm 43.5 months (Table 1).

\section{Risk factor analysis for distant metastasis}

Clinicopathologic factors related to distant metastasis are shown in Table 2. In univariate analysis, older patients had a tendency for distant metastasis with a 45-year cut-off age being statistically significant. Patients with distant metastasis were linked to vascular invasion and widely invasive FTC. Tg levels, which was checked in patients underwent total thyroidectomy in the first postoperative month, were higher in the distant metastasis group. Five patients with high Tg level had synchronous distant metastatic lesions. Multivariate analysis indicated that old age and vascular invasion were statistically significant factors for distant metastasis.

\section{Eight patients with distant metastases}

The characteristics of the 8 patients with distant metastases: 5 with synchronous and 3 with metachronous metastasis, are described in Table 3. While they all still had the disease at the time of their final visit, none had succumbed.

Case 1

A femoral bone mass was discovered in a patient diagnosed with renal cell carcinoma. It was revealed to be a metastatic FTC. A total thyroidectomy and RAI therapy were performed. ${ }^{131} \mathrm{I}$ 
WBS showed iodine uptake at the left scapula, left humeral shaft, around the anterior arc of left 3rd rib, sacrum, left ilium, and left femur. As bone metastasis progressed, despite two additional high-dose RAI therapies, radiation treatment (RT) was performed with the kinase inhibitor, lenvatinib.

\section{Case 2}

Thyroid cancer was found in a patient during a cervical (C) spine metastatic lesion work-up. A metastatic FTC was found on C5 during the spine biopsy and a total thyroidectomy and RAI therapy were performed. Since left scapular uptake was seen on ${ }^{131}$ I WBS, high-dose RAI therapy was repeated. Cervical bone RT was performed with the kinase inhibitor, sorafenib, due to uptake in multiple cervical vertebrae.

\section{Case 3}

A patient's first high dose RAI ablation showed multiple thoracic-lumbar (T-L) spine and rib metastases. As RAI avidity was seen on the 1st and 2nd ${ }^{131} \mathrm{I}$ WBS, a 3rd RAI was planned.

Table 1. Clinicopathologic characteristics of follicular thyroid cancer patients $(n=149)$

\begin{tabular}{|c|c|}
\hline Characteristics & Value \\
\hline Age at diagnosis (yr) & $44.8 \pm 14.4(19-84)$ \\
\hline$<45$ & $78(52.3)$ \\
\hline$<55$ & $113(75.8)$ \\
\hline $\operatorname{Sex}(F: M)$ & 123:26 (82.6:17.4) \\
\hline Size $(\mathrm{cm})$ & $3.44 \pm 1.77(0.1-10.0)$ \\
\hline$>4$ & $48(32.2)$ \\
\hline Capsule invasion & $146(98.0)$ \\
\hline Vascular invasion & $53(35.6)$ \\
\hline ETE & $2(1.3)$ \\
\hline $\mathrm{RM}(+)$ & $2(1.3)$ \\
\hline Minimal invasive:widely invasive & $134: 15$ (89.9:10.1) \\
\hline Lymph node metastasis & $2(1.3)$ \\
\hline Multifocality & $5(3.4)$ \\
\hline \multicolumn{2}{|l|}{ T stage } \\
\hline $\mathrm{T} 1$ & $35(23.5)$ \\
\hline $\mathrm{T} 2$ & $65(43.6)$ \\
\hline T3 & $48(32.2)$ \\
\hline T4 & $1(0.7)$ \\
\hline \multicolumn{2}{|l|}{ N stage } \\
\hline NO & $147(98.7)$ \\
\hline N1 & $2(1.3)$ \\
\hline \multicolumn{2}{|l|}{ M stage } \\
\hline MO & $144(96.6)$ \\
\hline M1 & $5(3.4)$ \\
\hline \multicolumn{2}{|l|}{ TNM stage } \\
\hline I & $131(87.9)$ \\
\hline II & $14(9.4)$ \\
\hline IV & $4(2.7)$ \\
\hline Extent of surgery (less than TT:TT) & $87: 62(58.4: 41.6)$ \\
\hline RAI Tx & $43(28.9)$ \\
\hline \multicolumn{2}{|l|}{ Distant metastasis } \\
\hline Synchronous & $5(3.4)$ \\
\hline Metachronous & $3(2.0)$ \\
\hline Follow-up duration (mon) & $65.4 \pm 43.5(2-176)$ \\
\hline
\end{tabular}

Values are presented as mean (range) or number (\%).

$\mathrm{ETE}$ = extrathyroidal extension; $\mathrm{RM}$ = resection margin; $\mathrm{TNM}$ = tumor-node-metastasis; $\mathrm{TT}$ = total thyroidectomy; $\mathrm{RAI}=$ radioactive iodine. 
Table 2. Risk factors for distant metastasis

\begin{tabular}{|c|c|c|c|c|c|}
\hline \multirow[t]{2}{*}{ Characteristics } & \multicolumn{2}{|c|}{ No. (\%) } & \multirow[t]{2}{*}{$\mathrm{P}$} & \multicolumn{2}{|c|}{ Multivariate analysis } \\
\hline & Distant metastasis (+) & Distant metastasis (-) & & Hazard ratio $(95 \% \mathrm{Cl})$ & $\mathrm{P}$ \\
\hline Age & $58.9 \pm 12.6$ & $44.0 \pm 14.1$ & 0.004 & $1.068(1.012-1.126)$ & 0.016 \\
\hline$<45$ & $1(12.5)$ & $77(54.6)$ & 0.028 & n.a. & \\
\hline$<55$ & $4(50.0)$ & $109(77.3)$ & 0.097 & n.a. & \\
\hline Gender & $6: 2(75.0: 25.0)$ & $117: 24$ (83.0:17.0) & 0.629 & n.a. & \\
\hline Size $(\mathrm{cm})$ & $3.66 \pm 1.81$ & $3.43 \pm 1.77$ & 0.717 & n.a. & \\
\hline$>4$ & $3(37.5)$ & 45 (31.9) & 0.713 & n.a. & \\
\hline Capsule invasion & $8(100.0)$ & $138(97.9)$ & 1.000 & n.a. & \\
\hline Vascular invasion & $7(87.5)$ & $46(32.6)$ & 0.003 & $8.459(1.577-45.366)$ & 0.013 \\
\hline ETE & $1(12.5)$ & $1(0.7)$ & 0.105 & n.a. & \\
\hline $\mathrm{RM}(+)$ & $1(12.5)$ & $1(0.7)$ & 0.105 & n.a. & \\
\hline Widely invasive & $4(50.0)$ & $11(7.8)$ & 0.004 & $2.582(0.459-14.520)$ & 0.282 \\
\hline Lymph node metastasis & $0(0.0)$ & $2(1.4)$ & 1.000 & n.a. & \\
\hline Multifocality & $0(0.0)$ & $5(3.5)$ & 1.000 & n.a. & \\
\hline T stage $(1 / 2 / 3 / 4)$ & 2/3/3/0 (25.0/37.5/37.5/0.0) & $33 / 62 / 45 / 1(23.4 / 44.0 / 31.9 / 0.7)$ & 0.559 & n.a. & \\
\hline Extent of surgery (TT) & $5(62.5)$ & $57(40.4)$ & 0.278 & n.a. & \\
\hline $\operatorname{Tg}(\mathrm{ng} / \mathrm{mL})^{*}$ & $196.6 \pm 129.7$ & $38.6 \pm 85.1$ & $<0.001$ & n.a. & \\
\hline $\operatorname{Tg}-\mathrm{Ab}(\mathrm{U} / \mathrm{mL})^{*}$ & $0.3 \pm 0.2$ & $5.4 \pm 17.5$ & 0.521 & n.a. & \\
\hline Follow-up duration (mon) & $52.3 \pm 47.1$ & $66.1 \pm 43.3$ & 0.381 & n.a. & \\
\hline
\end{tabular}

Values are presented as mean (range) or number (\%).

$\mathrm{Cl}=$ confidence interval; $\mathrm{ETE}=$ extrathyroidal extension; $\mathrm{RM}=$ resection margin; $\mathrm{Tg}$ = thyroglobulin; Tg-Ab = thyroglobulin-antibody; $\mathrm{TT}=$ total thyroidectomy; n.a. $=$ not available.

${ }^{*}$ Checked in patients underwent TT in postoperative 1 month.

Table 3. Eight patients with distant metastasis

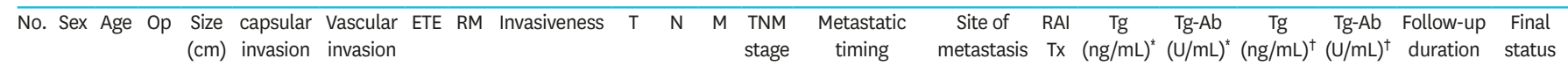

\begin{tabular}{|c|c|c|c|c|c|c|c|c|c|c|c|c|c|c|c|c|c|c|c|c|c|c|}
\hline & & & & & & & & & & & & & & & & & & & & \multicolumn{3}{|c|}{ (mon) } \\
\hline 1 & $M$ & 66 & $\mathrm{TT}$ & 1.9 & $(+)$ & $(+)$ & $(-)$ & $(-)$ & Minimally & 1 & 0 & 1 & 4 & Synchronous & Bone & $(+)$ & 250.00 & 0.15 & 250.00 & 0.23 & 16 & $\begin{array}{c}\text { Alive } \\
\text { with ds }\end{array}$ \\
\hline 2 & $\mathrm{~F}$ & 67 & $\mathrm{TT}$ & 3.5 & $(+)$ & $(+)$ & $(-)$ & $(-)$ & Widely & 2 & 0 & 1 & 4 & Synchronous & Bone & $(+)$ & 361.98 & 0.14 & 450.00 & 0.11 & 82 & $\begin{array}{c}\text { Alive } \\
\text { with ds }\end{array}$ \\
\hline 3 & $F$ & 46 & $\mathrm{TT}$ & 2.2 & $(+)$ & $(+)$ & $(-)$ & $(-)$ & Minimally & 2 & 0 & 1 & 2 & Synchronous & Bone & $(+)$ & 172.46 & 0.45 & 49.45 & 0.17 & 14 & $\begin{array}{c}\text { Alive } \\
\text { with ds }\end{array}$ \\
\hline 4 & M & 68 & $\mathrm{TT}$ & 4.5 & $(+)$ & $(+)$ & $(-)$ & $(-)$ & Widely & 3 & 0 & 1 & 4 & Synchronous & Bone & $(+)$ & 193.10 & 0.19 & 331.30 & 0.18 & 23 & $\begin{array}{c}\text { Alive } \\
\text { with ds }\end{array}$ \\
\hline 5 & $\mathrm{~F}$ & 78 & $\mathrm{TT}$ & 6.4 & $(+)$ & $(+)$ & $(+)$ & $(+)$ & Widely & 3 & 0 & 1 & 4 & Synchronous & Lung & $(-)$ & 5.66 & 0.53 & 9.56 & 0.04 & 15 & $\begin{array}{c}\text { Alive } \\
\text { with ds }\end{array}$ \\
\hline 6 & $\mathrm{~F}$ & 51 & $\mathrm{RL}$ & 3.0 & $(+)$ & $(+)$ & $(-)$ & $(-)$ & Minimally & 2 & 0 & 0 & 1 & Metachronous & Lung & $(-)$ & 23.40 & 1.56 & 2.90 & 0.25 & 150 & $\begin{array}{c}\text { Alive } \\
\text { with ds }\end{array}$ \\
\hline 7 & $\mathrm{~F}$ & 42 & $\mathrm{RL}$ & 6.0 & $(+)$ & $(-)$ & $(-)$ & $(-)$ & Minimally & 3 & 0 & 0 & 1 & Metachronous & Lung & $(-)$ & 10.87 & 2.95 & 1.61 & 5.35 & 56 & $\begin{array}{c}\text { Alive } \\
\text { with ds }\end{array}$ \\
\hline 8 & $F$ & 53 & LL & 1.8 & $(+)$ & $(+)$ & $(-)$ & $(-)$ & Widely & 1 & 0 & 0 & 1 & Metachronous & Bone & $(-)$ & 8.16 & 0.24 & 2.14 & 0.25 & 63 & $\begin{array}{c}\text { Alive } \\
\text { with ds }\end{array}$ \\
\hline
\end{tabular}

$\mathrm{Op}=$ operation; $\mathrm{TT}$ = total thyroidectomy; $\mathrm{RL}=$ right thyroid lobectomy; $\mathrm{LL}=$ left thyroid lobectomy; $\mathrm{TNM}=$ tumor-node- metastasis; $\mathrm{ETE}=\mathrm{extrathyroidal}$ extension; $\mathrm{RM}$ = resection margin; $\mathrm{RAI}$ = radioactive iodine; $\mathrm{Tg}$ = thyroglobulin; $\mathrm{Tg}-\mathrm{Ab}=$ thyroglobulin-antibody; $\mathrm{ds}=\mathrm{disease}$.

${ }^{*}$ Checked in patients underwent TT in postoperative 1 month; ${ }^{\dagger}$ Checked in patients underwent TT in postoperative 3 months.

Case 4

Right posterior 8th rib metastasis was found on a patient's first high-dose RAI ablation. As a left humeral uptake was observed during the 2nd RAI therapy, resection of the rib lesion was performed. A 3rd RAI therapy was scheduled.

\section{Case 5}

A patient had a large widely invasive FTC with gross muscle invasion. Although multiple pulmonary metastases were seen on her postoperative chest CT, RAI therapy could not be performed because of her psychiatric illness. 


\section{Case 6}

After a patient underwent a right thyroid lobectomy during her first operation, a Tg level increase up to $240.78 \mathrm{ng} / \mathrm{mL}$ was detected on postoperative month 146 while under TSH suppression. As a chest CT showed multiple pulmonary metastatic nodules, completion thyroidectomy was performed. RAI therapy was planned.

\section{Case 7}

A patient's chest CT showed multiple pulmonary metastases in postoperative month 34 . $\mathrm{Tg}$ levels rose to $34.47 \mathrm{ng} / \mathrm{mL}$ under TSH suppression. A completion thyroidectomy and two cycles of high-dose RAI therapy were performed. However, persistent lung metastases were seen on ${ }^{131}$ I WBS.

\section{Case 8}

A patient had a left thyroid lobectomy. Although a 1.8-cm widely invasive FTC was revealed on pathologic examination, completion thyroidectomy was not performed because of its small size and patient refusal. Follow-up PET-CT scans showed multiple skull, rib, T-L spine, ischium, and femoral bone metastases in postoperative month 41. Tg levels increased to $52.38 \mathrm{ng} / \mathrm{mL}$. Completion thyroidectomy and 3 high doses of RAI therapy were performed. However, lesion progression into the thoracic spine and ischium were detected.

\section{Clinicopathologic factors associated with vascular invasion}

There were more male patients with vascular invasion than female patients $(26.4 \%$ [14/53] vs. $12.5 \%$ [12/96], $\mathrm{P}=0.042)$. Patients with vascular invasion had larger tumors than patients without vascular invasion $(4.18 \pm 1.65 \mathrm{~cm}$ vs. $3.04 \pm 1.71 \mathrm{~cm}, \mathrm{P}<0.001)$. The proportion of tumors $>4 \mathrm{~cm}$ was $49.1 \%$ (26/53) for patients with vascular invasion vs. $22.9 \%$ (22/96) for patients without vascular invasion $(\mathrm{P}=0.002)$. $\mathrm{T}$ stage was more advanced in patients with vascular invasion $(\mathrm{P}<0.001)$. Distant metastasis occurred more commonly in patients with vascular invasion than in those without vascular invasion $(13.2 \%$ [7/53] vs. $1.0 \%$ [1/96], $\mathrm{P}=0.003)$. Synchronous distant metastasis was detected only in patients with vascular invasion $(9.4 \%$ [5/53], $\mathrm{P}=0.005)$. However, metachronous distant metastasis occurred to the same extent in both groups $(3.8 \%[2 / 53]$ vs. $1.0 \%$ [1/96], $\mathrm{P}=0.288)$.

\section{DISCUSSION}

In this study, old age and vascular invasion were significant factors for distant metastasis in FTC. Univariate analysis showed that age $\geq 45$ years and widely invasive FTC were associated with distant metastasis. Tg levels in the first postoperative month in patients with a total thyroidectomy were associated with synchronous distant metastasis in univariate analysis.

According to FTC studies with distant metastasis, old age ( $\geq 45$ years), larger tumor size ( $>4$ $\mathrm{cm}$ ), the presence of vascular invasion, extensive vascular invasion ( $\geq 4$ foci), incomplete resections, lymph node metastasis, and loco-regional recurrence were significantly associated with distant metastases $(9,11-16)$.

Survival rates of FTC patients with distant metastases were worse than for FTC patients without distant metastases. Synchronous distant metastasis was a strong prognostic factor for cause specific survival (CSS). Ten-year CSS rates of FTC patients with and without synchronous distant metastasis were $21 \%$ and $99 \%$, respectively (10). Five-year CSS rates 
for synchronous distant metastasis groups, metachronous distant metastasis groups, and groups with no distant metastasis were $58 \%, 80 \%$, and $100 \%$, respectively, and 10 -year CSS rates were $34 \%, 64 \%$, and $100 \%$, respectively. Groups with no distant metastasis showed significantly better survival than distant metastasis groups $(\mathrm{P}<0.001)$. Disease-specific deaths were found in $18 \%$ of patients in the distant metastasis group (7). Cumulative survival rate was significantly poorer in synchronous distant metastasis patients. Patients aged $\geq 45$ or $\geq 50$ years, tumor size $>4 \mathrm{~cm}$, combined capsular and vascular invasion, and of the male sex were linked to an increased risk of death from FTC $(17,18)$.

It would be advantageous to determine the adverse influence of synchronous or metachronous distant metastasis on the disease prognosis. Synchronous distant metastasis appears to show a more severe disease status in our study. Four out of five with synchronous distant metastasis had bone metastasis. Progression of the disease continued despite repeat RAI therapy and kinase inhibitor administration. In metachronous distant metastasis patients, only one suffered from bone metastasis, but also showed disease progression after RAI therapy. Because of the rarity of the disease, most of the previous studies did not distinguish between synchronous and metachronous distant metastasis. In one study, the authors claimed that these two groups had different disease entities, disease characteristics, and prognoses. Differentiated thyroid carcinoma patients presenting with initial distant metastasis appear to have relatively favorable outcomes compared with patients who developed distant metastasis after their initial treatment (19). However, this study dealt with all differentiated thyroid carcinomas including PTC. In one FTC study, synchronous and metachronous distant metastasis groups showed no significant difference in disease specific survival rates between the two groups $(\mathrm{P}=0.15)(7)$. There was no significant difference in mortality between those who presented with metastatic disease and those who developed metastasis during the follow-up period ( $33 \%$ vs. $50 \%, \mathrm{P}=0.61$ ) (20). We could not determine different adverse survival effects between synchronous and metachronous patients because all our patients survived.

In this study, bone metastasis occurred more frequently than lung metastasis. Interestingly, the synchronous distant metastasis group showed more frequent bone metastases while the metachronous distant metastasis group had more frequent lung metastases. In one study, 12 patients were diagnosed with metastatic disease at presentation, with bone being the most common site $(75 \%)$. In the remaining eight cases, metastases developed at a median of 4.5 years (range: 2-8 years) after initial thyroid surgery, with lungs being the most common site (50\%) (20). In another study, when 12 out of 132 patients $(9 \%)$ presented with distant metastases, they were present in the bone (5 patients), lungs (4 patients), pituitary gland (1 patient), ovary (1 patient), and scalp (1 patient) (21).

FTC metastasizes to bone more frequently than PTC. Only lung metastasis was more frequent in PTC than in FTC ( $79 \%$ vs. $13 \%, \mathrm{P}<0.001)$, and only bone metastasis was more frequent in FTC than in PTC ( $50 \%$ vs. $8 \%, \mathrm{P}<0.001)(6)$. The incidence of bone metastasis from thyroid carcinomas in a study of all thyroid carcinomas was $5.0 \%$; $45.4 \%$ had follicular, $36.3 \%$ had papillary, $6.8 \%$ had anaplastic, $6.8 \%$ had medullary, and 2 had Hürthle cell carcinomas (22).

Bone metastases can occur in vertebral bodies followed by the pelvis, femur, skull, and ribs. RAI therapy for patients with bone metastases is rarely curative, but some patients with RAI avid bone metastases may benefit from RAI therapy. Patients undergoing RAI therapy for bone metastases should also be considered for directed bone lesion therapy (23). This may 
include surgery, external beam RT, other focal treatment modalities, and systemic therapy with bone-directed agents. As bone metastases are less likely to concentrate radioiodine, and with high dose RAI therapy effective in only $55 \%$ of patients and remission in only $17 \%$ of patients, it is associated with a worse prognosis than pulmonary metastases. Patients are rarely cured, but they may experience a partial tumor response or disease stabilization with symptomatic improvement. External beam RT may be used for palliation (4). Therefore, detection of bone metastasis in FTC at diagnosis or during follow-up is very important when managing FTC patients.

The presence of vascular invasion was a significant factor for distant metastasis in this study and was an independent risk factor for distant metastasis when using multivariate analyses $(12,16)$. It was the most powerful predictor of distant metastasis after multivariate linear regression with old age (24). Patients who had vascular invasion with or without capsular invasion had a less favorable prognosis compared with patients who only had capsular invasion (21). According to the most recent World Health Organization (WHO) guidelines, extensive angioinvasion is associated with a worse prognosis although vascular invasion alone is not a component of diagnosis for widely invasive FTC (3). Vascular invasion and extensive vascular invasion were also emphasized in the 2015 American Thyroid Association (ATA) guidelines (23). The pathological parameters emphasized by the 2015 ATA guidelines include: aggressive histology, capsular invasion, the presence and extent of vascular invasion, extra-thyroidal extension, the number of lymph nodes with metastatic disease, and the size of metastatic foci. Therefore, the presence of vascular invasion is a very important prognostic factor for FTC. It is increasingly important for pathologists to report the presence and extent of vascular invasion to help clinicians understand their potential impact on patient management.

Three patients with metachronous distant metastasis underwent lobectomy during their first operation. However, the extent of the operation did not affect distant recurrence after analyzing metachronous distant metastasis in 144 cases $(3.4 \%$ [3/87] in less than total thyroidectomy vs. $0.0 \%[0 / 57]$ in total thyroidectomy, $\mathrm{P}=0.278)$. In other studies, the surgical extent did not have an influence on the development of distant metastases during followup using multivariate analysis $(12,25)$.

This study had several limitations. First, the mean follow-up period (66 months) was relatively short. However, more than half had a follow-up period of over 60 months and it was over 36 months in $67 \%$ of the cases. Metachronous metastasis occurred in postoperative months 34,41 , and 146 . Secondly, we had a small number of FTC patients resulting in a corresponding small number of distant metastasis cases. We could not analyze respective characteristics of synchronous and metachronous distant metastasis.

\section{CONCLUSION}

In FTC, old age and vascular invasion were significant factors for distant metastasis. Univariate analysis indicated that old age (especially, $\geq 45$ years), vascular invasion, widely invasive FTC, and $\mathrm{Tg}$ in the first postoperative month (synchronous distant metastasis) were associated with distant metastasis. During the care of FTC patients, it is important to predict distant metastasis. Bone metastasis was frequent in FTC, and it was difficult to cure. Bone and lung metastatic work-ups should be performed routinely to discover distant metastatic lesions. 


\section{REFERENCES}

1. Townsend CM Jr, Beauchamp RD, Evers BM, Mattox KL. Sabiston Textbook of Surgery: The Biological Basis of Modern Surgical Practice. 20th ed. Philadelphia (PA): Elsevier Saunders; 2016.

2. Korea Central Cancer Registry; National Cancer Center (KR); Ministry of Health and Welfare (KR). Annual Report of Cancer Statistics in Korea in 2015. Goyang: Korea Central Cancer Registry; 2017.

3. Lloyd RV, Osamura RY, Klöppel G, Rosai J. WHO Classification of Tumours of Endocrine Organs. 4th ed. Lyon: International Agency for Research on Cancer; 2017.

4. McHenry CR, Phitayakorn R. Follicular adenoma and carcinoma of the thyroid gland. Oncologist 2011;16:585-93. PUBMED | CROSSREF

5. Sampson E, Brierley JD, Le LW, Rotstein L, Tsang RW. Clinical management and outcome of papillary and follicular (differentiated) thyroid cancer presenting with distant metastasis at diagnosis. Cancer 2007;110:1451-6. PUBMED | CROSSREF

6. Kim H, Kim HI, Kim SW, Jung J, Jeon MJ, Kim WG, et al. Prognosis of differentiated thyroid carcinoma with initial distant metastasis: a multicenter study in Korea. Endocrinol Metab (Seoul) 2018;33:287-95. PUBMED | CROSSREF

7. Shimbashi W, Sugitani I, Kawabata K, Mitani H, Toda K, Yamada K, et al. Thick tumor capsule is a valuable risk factor for distant metastasis in follicular thyroid carcinoma. Auris Nasus Larynx 2018;45:147-55. PUBMED | CROSSREF

8. Leite AK, Cavalheiro BG, Kulcsar MA, Hoff AO, Brandão LG, Cernea CR, et al. Deaths related to differentiated thyroid cancer: a rare but real event. Arch Endocrinol Metab 2017;61:222-7. PUBMED | CROSSREF

9. Sugino K, Ito K, Nagahama M, Kitagawa W, Shibuya H, Ohkuwa K, et al. Prognosis and prognostic factors for distant metastases and tumor mortality in follicular thyroid carcinoma. Thyroid 2011;21:751-7. PUBMED | CROSSREF

10. Ito Y, Hirokawa M, Masuoka H, Yabuta T, Kihara M, Higashiyama T, et al. Prognostic factors of minimally invasive follicular thyroid carcinoma: extensive vascular invasion significantly affects patient prognosis. Endocr J 2013;60:637-42. PUBMED | CROSSREF

11. Ju YW, Kim SJ, Chai YJ, Yi JW, Seong CY, Kim JK, et al. Clinicopathologic features in minimally invasive follicular thyroid cancer patients with distant metastasis. Korean J Endocr Surg 2016;16:100-6. CROSSREF

12. Sung MK, Lee YM, Sung TY, Yoon JH, Chung KW, Hong SJ. Risk factors for distant metastasis in patients with follicular thyroid carcinoma. Korean J Endocr Surg 2016;16:25-30. CROSSREF

13. Lee YM, Sung TY, Yoon JH, Chung KW, Hong SJ. Long term outcome of minimally invasive follicular thyroid carcinoma according to risk group stratification. J Endocr Surg 2018;18:183-90. CROSSREF

14. Kim WW, Hur SM, Kim SH, Lee SK, Kim S, Oh YL, et al. Prognostic factors and treatment in follicular thyroid carcinoma. J Korean Surg Soc 2010;78:149-56. CROSSREF

15. Lee YM, Song DE, Kim TY, Sung TY, Yoon JH, Chung KW, et al. Risk factors for distant metastasis in patients with minimally invasive follicular thyroid carcinoma. PLoS One 2016;11:e0155489. PUBMED | CROSSREF

16. Lee YM, Lee YH, Song DE, Kim WB, Sung TY, Yoon JH, et al. Prognostic impact of further treatments on distant metastasis in patients with minimally invasive follicular thyroid carcinoma: verification using inverse probability of treatment weighting. World J Surg 2017;41:138-45. PUBMED | CROSSREF

17. Sugino K, Kameyama K, Ito K, Nagahama M, Kitagawa W, Shibuya H, et al. Outcomes and prognostic factors of 251 patients with minimally invasive follicular thyroid carcinoma. Thyroid 2012;22:798-804. PUBMED | CROSSREF

18. Stenson G, Nilsson IL, Mu N, Larsson C, Lundgren CI, Juhlin CC, et al. Minimally invasive follicular thyroid carcinomas: prognostic factors. Endocrine 2016;53:505-11. PUBMED | CROSSREF

19. Lee J, Soh EY. Differentiated thyroid carcinoma presenting with distant metastasis at initial diagnosis clinical outcomes and prognostic factors. Ann Surg 2010;251:114-9. PUBMED | CROSSREF 
20. Parameswaran R, Shulin Hu J, Min En N, Tan WB, Yuan NK. Patterns of metastasis in follicular thyroid carcinoma and the difference between early and delayed presentation. Ann R Coll Surg Engl 2017;99:151-4. PUBMED | CROSSREF

21. D'Avanzo A, Treseler P, Ituarte PH, Wong M, Streja L, Greenspan FS, et al. Follicular thyroid carcinoma: histology and prognosis. Cancer 2004;100:1123-9.

PUBMED | CROSSREF

22. Wu K, Hou SM, Huang TS, Yang RS. Thyroid carcinoma with bone metastases: a prognostic factor study. Clin Med Oncol 2008;2:129-34.

PUBMED | CROSSREF

23. Haugen BR, Alexander EK, Bible KC, Doherty GM, Mandel SJ, Nikiforov YE, et al. 2015 American Thyroid Association management guidelines for adult patients with thyroid nodules and differentiated thyroid cancer: the American Thyroid Association guidelines task force on thyroid nodules and differentiated thyroid cancer. Thyroid 2016;26:1-133.

PUBMED | CROSSREF

24. O'Neill CJ, Vaughan L, Learoyd DL, Sidhu SB, Delbridge LW, Sywak MS. Management of follicular thyroid carcinoma should be individualised based on degree of capsular and vascular invasion. Eur J Surg Oncol 2011;37:181-5. PUBMED | CROSSREF

25. Goffredo P, Cheung K, Roman SA, Sosa JA. Can minimally invasive follicular thyroid cancer be approached as a benign lesion?: a population-level analysis of survival among 1,200 patients. Ann Surg Oncol 2013;20:767-72.

PUBMED | CROSSREF 